to usual psychiatric treatment for bipolar disorders. International Journal of Neuropsychiatry, in press.

J. Scott Department of Psychological Medicine, PO Box 96, Institute of Psychiatry, De Crespigny Park, London SE5 8AF, UK. E-mail: j.scott@iop.kcl.ac.uk

E. Paykel Department of Psychiatry, University of Cambridge, UK

R. Morriss Department of Psychiatry, Royal Liverpool University Hospital, UK

R. Bentall Department of Psychology, University of Manchester, UK

P. Kinderman Department of Clinical

Psychology, University of Liverpool, UK

T. Johnson Medical Research Council Biostatistics Unit, Institute of Public Health, Cambridge, UK

R. Abbott, H. Hayhurst Department of Psychiatry, University of Cambridge, Addenbrooke's Hospital, Cambridge, UK

\section{Trial of risperidone in India - concerns}

The study by Khanna et al (2005) on the effectiveness of risperidone in acute mania raises many questions.

Why was the study done? The authors do not indicate that existing treatments have limitations that led them to test risperidone as an alternative.

Why was a placebo used when an effective treatment exists? This is particularly worrisome because, as the authors state, acute mania can be life-threatening and carries an increased risk of suicide.

Patients undergoing psychiatric treatment are a vulnerable group. How did patients give informed consent during an episode of acute mania?

Where were the trial sites? Who were the participants and what quality of care did they receive? What were the adverse events? How were seven participants from the placebo group lost to follow-up?

Regarding the 'wash-out' period before the trial, is it medically and morally justified to withhold treatment from patients during an episode of illness in intensive care?

Four authors state that they are drug company employees. Do the other authors have any competing interest to declare?

In what sense was the trial conducted according to the Declaration of Helsinki? Why do the authors mention the Declaration as revised in 1989, rather than a more recent revision?
We suggest that this trial could not have been conducted in a high-income country but may have been conducted in India because regulatory requirements could be fulfilled there. The use of a placebo when an effective treatment exists - and other elements of the study as mentioned above - goes against the Helsinki guidelines and those of the Indian Council of Medical Research (2000). Finally, publication of such studies in a leading journal such as the British Journal of Psychiatry gives credibility to unethical medical research and practice and is a matter of serious concern.

\section{Declaration of interest}

The authors are editors of the Indian Journal of Medical Ethics and have previously written or spoken against certain drug company practices, including sponsored research.

Indian Council of Medical Research (2000) Ethical Guidelines for Biomedical Research on Human Subjects. http: / /icmr. nic. in/ethical.pdf

Khanna, S., Vieta, E., Lyons, B., et al (2005)

Risperidone in the treatment of acute mania: doubleblind, placebo-controlled study. British Journal of Psychiatry, 187, 229-234.

S. Srinivasan Indian Journal of Medical Ethics, 8 Seadoll, 54 Chimbai Road, Bandra (W), Mumbai 400 050, India. E-mail: sandhyasrinivasan@vsnl.com

S. A. Pai Manipal Hospital, Bangalore, Mumbai, India

A. Bhan, A. Jesani Centre for Studies in Ethics and Rights, Mumbai, India

G.Thomas Railway Hospital, Chennai, India

Although it is encouraging to see the Journal take an active role in redressing 'editorial racism' as discussed in a previous editorial (Tyrer, 2005), there is a need to ensure that promotion of positive discrimination does not exacerbate the problem.

We feel that a recently published randomised double-blind placebo-controlled trial of risperidone performed in India illustrates the dangers inherent in such a policy (Khanna et al, 2005). The report had a number of serious shortcomings, which included omission of crucial details of the process of randomisation, interrater reliability and the measures taken to ensure masking. However, the most worrying aspect of the trial was the use of a placebo in the control group and the apparent absence of any ethical approval to proceed with this study. What was the justification for denying severely unwell and vulnerable patients access to appropriate treatment? Why was there no discussion about the ethical dilemmas associated with this study?

We support the Journal policy of combating editorial racism by promoting positive discrimination in the instructions to referees. However, the Journal must not relinquish its responsibilities as the official journal of the Royal College of Psychiatrists by failing to act as final arbiter for the quality (including the ethics) of the Journal's content.

Khanna, S., Vieta, E., Lyons, B., et al (2005) Risperidone in the treatment of acute mania: doubleblind, placebo-controlled study. British Journal of Psychiatry, 187, 229-234.

Tyrer, P. (2005) Combating editorial racism in psychiatric publications. British Journal of Psychiatry, $\mathbf{1 8 6}$ I-3.

A. Murtagh Royal College of Surgeons in Ireland, Education and Research Centre, Beaumont Hospital, Dublin 9, Ireland. E-mail: amurtagh@rcsi. ie

K. C. Murphy Department of Psychiatry, Royal College of Surgeons in Ireland, Education and Research Centre, Beaumont Hospital, Dublin, Ireland

With a sample size of 290 patients the report by Khanna et al (2005) buttresses the data about efficacy of atypical antipsychotics in the treatment of acute mania, but the article also raised the following concerns.

One of the sites had to be withdrawn from the study after enrolling three participants because of concerns about data quality. However, the data from these individuals were still included in the safety analyses. We are of the opinion that if there were concerns about the data from one particular site, then that site should have been excluded from any further analyses.

We also have concerns about the legitimacy and validity of the informed consent obtained from 145 patients with acute mania and a mean Young Mania Rating Scale score of 37.5 to be enrolled in the placebo arm of a clinical trial. Article 4 of the World Medical Association Declaration of Helsinki (World Medical Association, 1989) states that biomedical research involving human participants cannot legitimately be carried out unless the 